Reprod. Nutr. Dévelop., 1988, 28 (6 B), 1781-1790

\title{
Les échecs du développement embryonnaire
}

\author{
Michelle PLACHOT, Anne-Marie JUNCA, Jacqueline MANDELBAUM, \\ J. COHEN $\left({ }^{*}\right)$, J. SALAT-BAROUX $\left({ }^{* *}\right)$
}

U 173 INSERM Groupe de Recherches sur la Fécondation in vitro, Hôpital Necker-Enfants-Malades, 149, rue de Sèvres, 75743 Paris Cedex 15.

(*) Service de Gynécologie Obstétrique, Hôpital de Sèvres, 141. Grande-Rue, 92310 Sèvres.

$\left(^{* *}\right)$ Maternité Guy-Le-Lorier,

Hopital Tenon, 4, rue de la Chine, 75020 Paris.

\section{Summary. Embryonic development failures.}

Abnormal embryo development represents the major cause of implantation failures and accounts for the low rate of human fertility in vivo or in vitro. Chromosome abnormalities are widely involved in this process as $26 \%$ of oocytes, $8 \%$ of fertilizing spermatozoa and $29 \%$ of preimplantation embryos carry a chromosome aberration induced by meiotic (aneuploidy) or mitotic (mosaic) non disjunctions. Fertilization anomalies (possibly increased by in vitro procedures) were recorded: $1.6 \%$ of embryos resulted from parthenogenesis and $6.4 \%$ were polyploid (mainly polyspermic).

A morphological, histological and ultrastructural study of embryos recovered after in vivo or in vitro fertilization showed some anomalies: multinucleated blastomeres, cytoplasmic fragments in the perivitelline space, vacuoles, associated or not with developmental impairement. Finally, a few embryos appeared to be free of abnormalities.

The analysis of in vitro developmental capacities of normal or abnormal embryos showed great differences: parthenones exceptionally reached the blastocyste stage and therefore probably did not implant. The diploid embryos used in this study were (for ethical reasons) more or less fragmented and gave evidence of low developmental capacities, limited to the 3rd cleavage. Triploid embryos were able to further develop as some of them reached the early blastocyste stage; they represented the major cause of chromosomal 1 st trimester abortions. It is interesting to note that $47 \%$ of tripronucleated ova divided directly into 3 and 6 cells (probably via a tripolar spindle) instead of 2 and 4 cells as classically described. Finally, tetraploid embryos expressed a precocious lethality as none developed beyond the 3rd cleavage.

To conclude, many embryos carry genetic and/or cytological abnormalities which may be enhanced by superovulation treatments. The selection proceeds through all pre-and postimplantation steps, and as a matter of fact no more than $0.6 \%$ newborns are abnormal.

II est aujourd'hui clairement établi que la grande majorité des embryons meurent en tout début de grossesse puisque $70 \%$ d'entre eux ont cessé leur développement à la fin du premier trimestre. Les raisons de cette mortalité embryonnaire sont variées dans la mesure où des facteurs embryonnaires intrinsèques et/ou des facteurs maternels peuvent être impliqués. La connaissance des mécanismes liés à la mortalité embryonnaire a fait des progrès

Reproduction, Nutrition, Développement, $n^{\circ} 6 \mathrm{~B} / 88-17$ 
considérables depuis le développement de la fécondation in vitro. L'étude de la qualité de l'ovocyte, des anomalies de la fécondation, des capacités de développement des embryons pendant la période pré- et postimplantatoire a éclairé d'un jour nouveau un processus jusqu'alors se déroulant à l'abri de toute investigation scientifique.

\section{Les anomalies méiotiques.}

Chez l'homme, les anomalies chromosomiques représentent la raison majeure de la mortalité embryonnaire pré- et postimplantatoire. La FIV offre la possibilité d'étudier le caryotype des ovocytes obtenus après superovulation. En raison de la rareté de l'ovocyte humain expérimental, seulement deux études rapportent le taux d'anomalies chromosomiques des ovocytes humains au recueil : il est de $34 \%$ (Martin et al., 1986) à $50 \%$ sur 73 ovocytes (Wramsby et al., 1987).

En revanche, l'analyse chromosomique des ovocytes non fécondés, $42 \mathrm{~h}$ après l'insémination est aisée. Nous avons étudié le caryotype de 316 ovocytes au stade de métaphase II (Plachot et al., 1988c).

Vingt-six pour cent présentaient une anomalie chromosomique :- - soit une hypohaploïdie (moins de 23 chromosomes avec de 1 à 5 chromosomes manquants essentiellement des groupes B, C ou D) ; - soit une hyperhaploïdie (plus de 23 chromosomes avec de 1 à 5 chromosomes surnuméraires pour les mêmes groupes de chromosomes) ; - soit une anomalie de structure avec une délétion des bras longs d'un chromosome $\mathrm{G}$; - soit une diploïdie, mettant en évidence une polyploïdie au stade des ovogonies, ou une anomalie du processus méiotique.

Dans la littérature, le taux d'anomalies chromosomiques varie de 11 à $27 \%$. Si l'on compare ces chiffres à ceux des autres espèces de mammifères ( 1 à $10 \%$ d'aneuploïdie) ou bien aux spermatozoïdes humains fécondants ( $8 \%$ d'aneuploidie), le taux d'anomalies dans les ovocytes humains semble très élevé.

Pour tenter d'évaluer l'influence de certains paramètres cliniques sur la survenue des anomalies chromosomiques, le taux d'aneuploïdie a été calculé en fonction de: l'indication de la FIV, l'âge maternel, le type de traitement de stimulation et les doses de gonadotrophines.

// n'a été rapporté aucun effet :

- de l'indication tubaire, idiopathique, masculine, endométriose ;

- du type de traitement de stimulation (Clomid/hMG, hMG seul, analogues du LH-RH/hMG) :

- des doses de gonadotrophines administrées (6 à 46 ampoules de FSH ou hMG par cycle).

En revanche, nous avons observé une augmentation du taux de nondisjonctions chez les femmes de plus de 35 ans (38\% vs $24 \%$ chez les femmes de moins de 35 ans) confirmant le rôle prépondérant de l'âge maternel dans les anomalies méiotiques.

L'utilisation des techniques de dénaturation chromosomique a permis dans certains cas de déterminer l'origine de l'anomalie. C'est ainsi que $60 \%$ de toutes les trisomies surviennent par non-disjonction au cours de la $1^{\text {re }}$ division méiotique 
de l'ovocyte (par malségrégation des chromosomes maternels dans l'ovocyte et le globule polaire) et très peu au cours de la $2^{\mathrm{e}}$ division méiotique dans l'ovocyte ou le spermatozoïde. Il n'a été mis en évidence aucun effet de l'âge paternel.

\section{Les anomalies de la fécondation.}

A côté de ces anomalies purement méiotiques intervenant au niveau de l'ovocyte ou du spermatozoïde, les anomalies propres à la fécondation représentent une cause importante d'échec du développement. En effet, la présence de 2 pronuclei, 1 mâle et 1 femelle, est absolument indispensable pour un développement embryonnaire et fœtal normal. Des œufs de souris possédant seulement 2 pronuclei femelle (par transfert de noyaux) peuvent se développer jusqu'au stade de 25 somites mais possèdent très peu de tissu extra-embryonnaire.

De la même manière, des embryons présentant 2 pronuclei mâles conduisent à un retard de l'embryogenèse et ne vont jamais à terme, bien que le trophoblaste se développe mieux que dans le cas des embryons présentant 2 pronuclei femelle (Barton et al., 1984). Or, dans le cadre de la FIV humaine lorsque les ovocytes sont examinés $17 \mathrm{~h}$ après l'insémination au stade des pronuclei, 3 situations sont possibles en fonction du nombre de noyaux présents dans le cytoplasme ovocytaire.

2.1. La parthénogenèse. - Dans $1,6 \%$ des cas on observe un seul pronucleus et les embryons qui en résultent sont haploïdes (46\%) ou diploïdes ( $29 \%)$ selon qu'il y a eu ou non émission du $2^{\mathrm{e}}$ globule polaire. Le caryotype de ces embryons montrant toujours une formule chromosomique femelle témoigne qu'ils sont vraisemblablement la conséquence d'une activation parthénogénétique. De plus, dans $26 \%$ des cas, leur caryotype est aneuploïde. En effet, ces embryons présentent le même taux d'anomalies chromosomiques que les ovocytes dont ils proviennent directement sans évidemment aucune contribution paternelle. Les expériences réalisées chez les mammifères ont montré que ces embryons parthénogénétiques avaient une tendance à la diploïdisation spontanée au tout début de l'embryogenèse conduisant à la formation de mosaïques haploïde/diploïde, comme nous l'avons observé dans notre étude dans $25 \%$ des cas.

2.2. La fécondation normale. - Dans $92 \%$ des cas, on observe 2 pronuclei dans le cytoplasme ovocytaire le lendemain de l'insémination. Pour autant, seulement $78 \%$ des embryons qui résultent de ces fécondations apparemment normales sont diploïdes. Cinq pour cent sont haplö̈des (probablement après exclusion d'un des 2 pronuclei) et $10 \%$ sont triploïdes (si l'un des 2 pronuclei contient un stock double de chromosomes). II est à noter que $1 \%$ des spermatozoïdes humains sont diploïdes. Enfin $7 \%$ sont des mosaïques et résultent de non-disjonctions mitotiques au tout début de l'embryogenèse.

2.3. La trip/oiddie. - Dans $6,4 \%$ des cas, on observe 3 pronuclei ou plus. Or le caryotype des embryons qui en résultent ne montre que $45 \%$ de triploïdes. Dans $17 \%$ des cas il est diploïde et dans $8 \%$ haploïde, témoignant de 
l'élimination de 1 ou 2 pronuclei respectivement, conséquence probable d'un asynchronisme de développement des pronuclei. Un taux élevé de mosaïques haploïde/triploïde ou diploïde/triploïde (30\%) est également observé chez ces embryons. Un retard à la fécondation peut conduire chez les mammifères à une fécondation dispermique ou à la rétention du $2^{\circ}$ globule polaire, et dans les 2 cas à la formation d'un œuf triploïde. Ainsi, les expériences de FIV montrent que le taux d'embryons triploïdes augmente avec la durée de la préincubation des ovocytes avant l'insémination et passe de $3 \%$ ( $<2$ h de préincubation) à $7,2 \%$ ( $>$ à $2 \mathrm{~h}$ ) témoignant d'un échec de la réaction corticale chez ces ovocytes âgés et donc d'un mauvais blocage de la polyspermie. De plus, lorsque la fécondation est spontanément retardée avec un retard de $24 \mathrm{~h}$ à l'apparition des pronuclei, le taux d'anomalies chromosomiques (87\%) et le taux de mosaïques (30\%) sont considérablement augmentés comparé aux fécondations chronologiquement normales (29,2 \% et 10,6\% respectivement) (Plachot et al., 1988b).

Enfin et contrairement aux trisomies et monosomies, la triploïdie n'est pas liée à l'âge maternel. Les différentes formes de triploïdie évoluent différemment, et certains fœtus forment des môles hydatiformes partielles, spécialement ceux comportant 2 lots de chromosomes paternels.

\section{La qualité de l'embryon.}

Le concept de qualité de l'embryon est difficile à définir : un bon embryon doit permettre l'obtention d'un être viable à travers toutes les étapes pré- et postimplantatoires. De plus, peu d'informations sont disponibles concernant le développement des œufs fécondés in vivo.

3.1. Développement embryonnaire dans le tractus génital femelle. - Le développement de bon nombre d'embryons humains est perturbé in vivo.

Ainsi, sur 35 embryons recueillis par perfusion des trompes et de l'utérus, 11 présentaient des anomalies morphologiques: blastomères inégaux, fragments cytoplasmiques anucléés, cellules multinucléées ou désintégrées (Hertig et al., 1956 ; Pereda et Croxatto, 1978). De plus, chez 13 femmes fertiles ayant eu un lavage utérin pour don d'embryon, 6 ont donné 10 blastocystes au cours de 31 cycles d'insémination et 7 n'en ont donné aucun malgré 52 lavages (Sauer et al., 1987). Enfin, plusieurs embryons avaient une segmentation retardée, la majorité n'étant pas parvenue au stade de morula ou blastocyste $120 \mathrm{~h}$ après la fécondation (Buster et al., 1985). Il est bien sûr impossible de savoir quel aurait été le destin de chacun de ces embryons. La fécondation in vitro, au contraire, permet de connaître l'aptitude des embryons à s'implanter et à évoluer jusqu'à terme en fonction de leur aspect cytomorphologique.

3.2. Analyse morphologique des embryons. - Mille quatre- vingt-un embryons humains de 2 à 8 cellules ont été classés en 4 groupes en fonction de 2 critères (Plachot et al., 1987), la taille et la régularité des blastomères et la présence ou non de fragments cytoplasmiques:

- groupe 1 : blastomères égaux et réguliers (46\%);

- groupe 2 : blastomères égaux et réguliers mais présence de fragments (24\%);

- groupe 3 : blastomères inégaux (20\%) ;

— groupe 4 : blastomères inégaux et fragmentés (10\%). 
Si on élimine ce dernier groupe, on voit que $90 \%$ des embryons présentent un aspect morphologique variable mais toujours compatible avec un développement embryonnaire ultérieur, puisque $9,8 \%, 15,4 \%$ et 6,5\%, de grossesses ont été obtenues après replacement unique de 229 embryons des groupes 1, 2 et 3 respectivement. Aucune grossesse n'a été obtenue après replacement d'embryons du groupe 4.

L'analyse cytologique, par contre, rectifie cette vision optimiste de la qualité de l'embryon. En effet, $66 \%$ des embryons des groupes 1, 2 et 3, $93 \%$ de ceux du groupe 4, ainsi que tous les embryons bloqués, présentent des anomalies cytologiques.

L'anomalie la plus souvent rencontrée est la présence de blastomères multinucléés pouvant contenir jusqu'à 6 noyaux de diamètre inégal. Cinquante-sept pour cent des embryons des groupes 1, 2 et 3 manifestent cette anomalie dans environ $1 / 3$ des blastomères. Ce taux atteint $83 \%$ en moyenne pour les embryons bloqués ou du groupe 4 dans lesquels $2 / 3$ des blastomères sont multinucléés. Cela a été rapporté par d'autres auteurs tels que Trounson et Sathananthan (1984) qui observent que les embryons humains régulièrement segmentés au stade de 2 à 4 cellules sont cytologiquement normaux alors que les embryons bloqués, retardés ou triploïdes présentent des anomalies telles que présence de fragments, blastomères multinucléés et chromosomes dissociés des microtubules. Les embryons en dégénérescence montrent une vacuolisation intense des blastomères et une augmentation de l'activité lysosomiale. Cependant, il est possible dans un même embryon de trouver des blastomères normaux et anormaux.

II semble donc que mis à part les embryons manifestement dégénérés, l'aspect morphologique des embryons ne représente pas un critère suffisant pour juger de leurs potentialités de développement.

Chez le rat, Shalgi (1984) avait montré que les capacités de développement des embryons provenant d'ovocytes fécondés in vitro sont la moitié de ceux fécondés in vivo, bien que l'aspect morphologique des embryons soit identique.

3.3. Vitesse de division et capacités de développement. - Lorsqu'ils sont examinés environ $42 \mathrm{~h}$ après l'insémination les embryons sont le plus souvent au stade de 2 à 4 cellules. Certains ont commencé le $3^{e}$ clivage et présentent donc au moins 5 blastomères. Lorsqu'on analyse les capacités de développement de 229 embryons en fonction du stade auxquels ils sont parvenus au moment du replacement unique in utero on observe que 9,6\% des embryons ayant atteint le stade 2 cellules s'implantent, 9,1\% pour le stade $3-4$ cellules ( ${ }^{\mathrm{e}}$ clivage) et $17,6 \%$ pour les stades $>$ à 4 cellules ( $3^{e}$ clivage). On observe donc une tendance vers une meilleure qualité des embryons lorsqu'ils se développent rapidement sans cependant de signification statistique.

3.4. Viabilité embryonnaire. - Il est maintenant clairement démontré que les embryons humains ont des potentialités de développement différentes liées à leur qualité intrinsèque. Le replacement de plusieurs embryons augmente donc les potentialités de grossesse. Ainsi, le taux d'implantation passe de $8 \%$ pour 
1 embryon replacé à $21,4 \%$ pour 2 embryons, $38,5 \%$ pour 3 embryons et $39,4 \%$ pour 4 embryons et on atteint probablement là un plateau où interviennent de manière prépondérante les facteurs maternels.

Globalement, la viabilité embryonnaire peut s'exprimer indépendamment des facteurs maternels en ne tenant compte que des replacements ayant donné une grossesse. C'est ainsi que 160 grossesses ont été obtenues chez des patientes ayant eu en tout 480 embryons replacés. Cent quatre-vingt-dix-sept se sont implantés, conduisant à un taux de viabilité embryonnaire de $41 \%$.

3.5. Les anomalies chromosomiques. - Nous avons observé que le taux d'anomalies chromosomiques dans les embryons produits par FIV est en relation avec leur aspect morphologique. Les embryons des groupes 1,2 et 3 possèdent $21,4 \%$ d'anomalies alors que les embryons du groupe $4,32,6 \%$. Cela permet de calculer un taux global d'anomalies chromosomiques qui est de $29,2 \%$ dont:

- Embryons haploïdes $1,6 \%$

- Embryons diploïdes $92 \%$

* $15 \%$ morphologiquement anormaux et comprenant

$32,6 \%$ d'anomalies chromosomiques

* $85 \%$ morphologiquement normaux et comprenant $21,4 \%$ d'anomalies chromosomiques $16,7 \%$

- Embryons triploïdes

\section{Les capacités de développement embryonnaire.}

Les capacités de développement ont été analysées chez les embryons provenant de fécondations normales ou anormales (Plachot et al., 1988a). Au cours des 2 premiers jours après l'insémination, on observe que seulement $4 \%$ des ovocytes présentant 2 pronuclei ( $2 \mathrm{PN}$ ) ne se divisent pas, alors que ce taux atteint $23 \%$ et $18 \%$ pour les ovocytes à 1 et 3 pronuclei (PN) respectivement (tabl. 1).

TABLEAU 1

Développement des embryons provenant d'œufs à 1,2 ou 3 pronuclei.

\begin{tabular}{lrrrrrr}
\hline \multirow{2}{*}{$\begin{array}{c}\text { Nombre de } \\
\text { pronuclei } \\
\text { à } J_{1}\end{array}$} & $n$ & Non clivés & \multicolumn{5}{c}{ Qualité de l'embryon à $J_{2}$} \\
\cline { 4 - 7 } & & & \multicolumn{1}{c}{1} & \multicolumn{1}{c}{3} & \multicolumn{1}{c}{4} \\
\hline 1 & 35 & $8(23 \%)^{\mathrm{a}}$ & $5(14 \%)$ & $8(23 \%)$ & $8(23 \%)$ & $6(17 \%)^{\mathrm{d}}$ \\
2 & 179 & $8(4 \%)^{\mathrm{b}}$ & $43(24 \%)$ & $53(30 \%)$ & $52(29 \%)$ & $23(13 \%)^{\mathrm{e}}$ \\
3 & 68 & $12(18 \%)^{\mathrm{c}}$ & $20(29 \%)$ & $12(18 \%)$ & $22(32 \%)$ & $2(3 \%)^{\dagger}$ \\
\hline
\end{tabular}

Test $\chi_{2}: \begin{array}{ll}a-b \\ b-c\end{array} \quad p<0,01 ; \quad \begin{aligned} & \text { d-f } \\ & \text { e-f }\end{aligned} \quad p<0,05$ 
L'étude histologique des œufs bloqués montre dans ce cas, soit un développement asynchrone des pronuclei, soit des vacuoles cytoplasmiques ou encore des têtes de spermatozoïdes surnuméraires non décondensées dans le cytoplasme.

L'aspect morphologique des embryons est également différent : le plus faible taux d'embryons dégénérés correspond aux triploïdes (3\%) comparé aux parthénotes $(13 \%)$ et aux diploïdes $(17 \%)$.

La vitesse de clivage des embryons est un bon indicateur de leur qualité. Si l'on compare le stade de division auxquels sont parvenus les 3 types d'œufs à $\mathrm{J}_{2}$ (42 $\mathrm{h}$ après l'insémination), on constate que les embryons triploïdes sont apparemment les plus avancés puisque $29 \%$ d'entre eux ont au moins 5 cellules comparé à $11 \%$ et $15 \%$ pour les œufs à 1 PN et 2 PN respectivement (tabl. 2). L'observation toutes les heures de ces 3 types d'œufs jusqu'au premier clivage montre que cette accélération apparente du développement des œufs triploïdes vient du fait que $47 \%$ d'entre eux se divisent directement en 3 cellules et sont donc au stade 6 cellules au $2^{\mathrm{e}}$ clivage alors que les autres se divisent en 2 puis 4 cellules.

\section{TABLEAU 2}

Vitesse de clivage des embryons provenant d'œufs à 1, 2 ou 3 pronuclei.

\begin{tabular}{lrrrr}
\hline $\begin{array}{c}\text { Nombre de } \\
\text { pronuclei } \\
\text { à } J_{1}\end{array}$ & $n$ & 2 cellules & 3 ou 4 cellules & 5 à 8 cellules \\
\cline { 3 - 5 } & 27 & $6(22 \%)$ & $18(67 \%)$ & $3(11 \%)^{\mathrm{a}}$ \\
1 & 171 & $50(29 \%)$ & $95(56 \%)$ & $26(15 \%)^{\mathrm{b}}$ \\
2 & 56 & $7(13 \%)$ & $33(59 \%)$ & $16(29 \%)^{\mathrm{c}}$ \\
\hline
\end{tabular}

Test $\chi_{2}:$ a-c : NS,

b-c: $p<0,05$.

L'ensemble de ces observations nous a permis de proposer un modèle de développement des œufs à 3 pronuclei (fig. 1).

1) Les 3 pronuclei participent à la formation d'un fuseau bipolaire lequel produit 2 cellules triploïdes. De tels embryons se développent au maximum jusqu'au $1^{\text {er }}$ trimestre de grossesse.

2) Les 3 pronuclei participent à la formation d'un fuseau tripolaire lequel produit directement 3 cellules globalement diploïdes. Cependant, la migration anarchique des chromosomes aux pôles du fuseau conduit le plus souvent à une mosaïque.

3) Un des pronuclei est exclu du $1^{\text {er }}$ clivage. Les 2 pronuclei restants forment un fuseau bipolaire et donc 2 blastomères. Si le pronucleus exclu est surnuméraire, il y a régulation et formation d'un embryon a priori normal. 
MODFLE DE DEVFLOPPEMENT DES OFUFS A 3 PRONUCLEUS

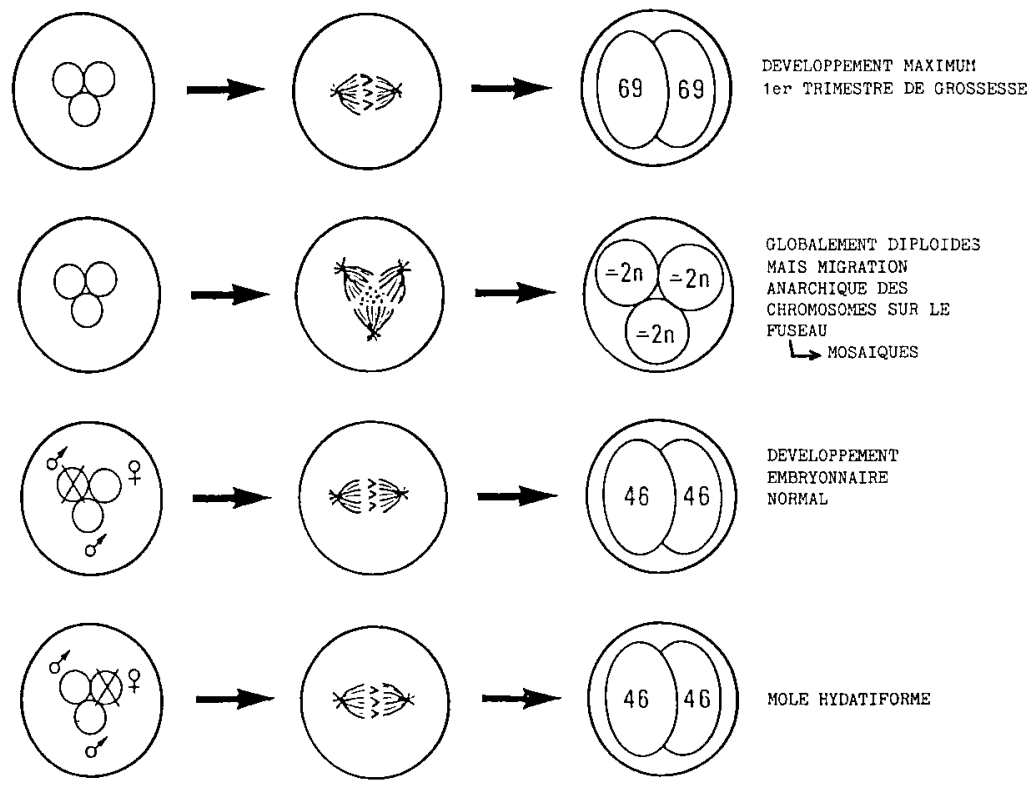

4) Si le pronucleus exclu n'est pas surnuméraire (pronucleus femelle en cas de dispermie) les 2 pronuclei mâles forment un fuseau bipolaire, puis 2 blastomères mais le développement de cet embryon formé sans contribution maternelle conduit à une môle hydatiforme.

Dans une étude préliminaire nous avons étudié la capacité de développement in vitro pendant 5 jours dans du $\mathrm{B}_{2}+15 \%$ de sérum de 76 embryons provenant d'œufs à 1, 2, 3 ou 4 pronuclei. La figure 2 montre que les embryons parthénogénétiques se développent activement jusqu'au $2^{\mathrm{e}}$ clivage, puis on note une diminution des potentialités de développement puisque très peu arrivent au stade de jeune blastocyste. Cela confirme l'observation selon laquelle aucun fotus haploïde ne parvient au stade du $1^{\text {er }}$ trimestre puisque n'ayant jamais été retrouvé ni dans les fausses couches spontanées ni à terme.

Les embryons provenant d'œufs à 2 pronuclei ont été sélectionnés (pour des raisons éthiques) comme étant surnuméraires après que 3 ou 4 embryons frais de bonne qualité aient été replacés, et de qualité morphologique insuffisante pour être congelés. Ils expriment effectivement cette qualité médiocre par une incapacité à franchir le $4^{\mathrm{e}}$ clivage.

Les embryons provenant d'œufs à 3 pronuclei sont les plus performants. On les retrouve au stade de jeunes blastocystes à $J_{5}$ postinsémination. En effet, ils sont une des raisons majeures des fausses couches d'origine chromosomique du $1^{\text {er }}$ trimestre. 


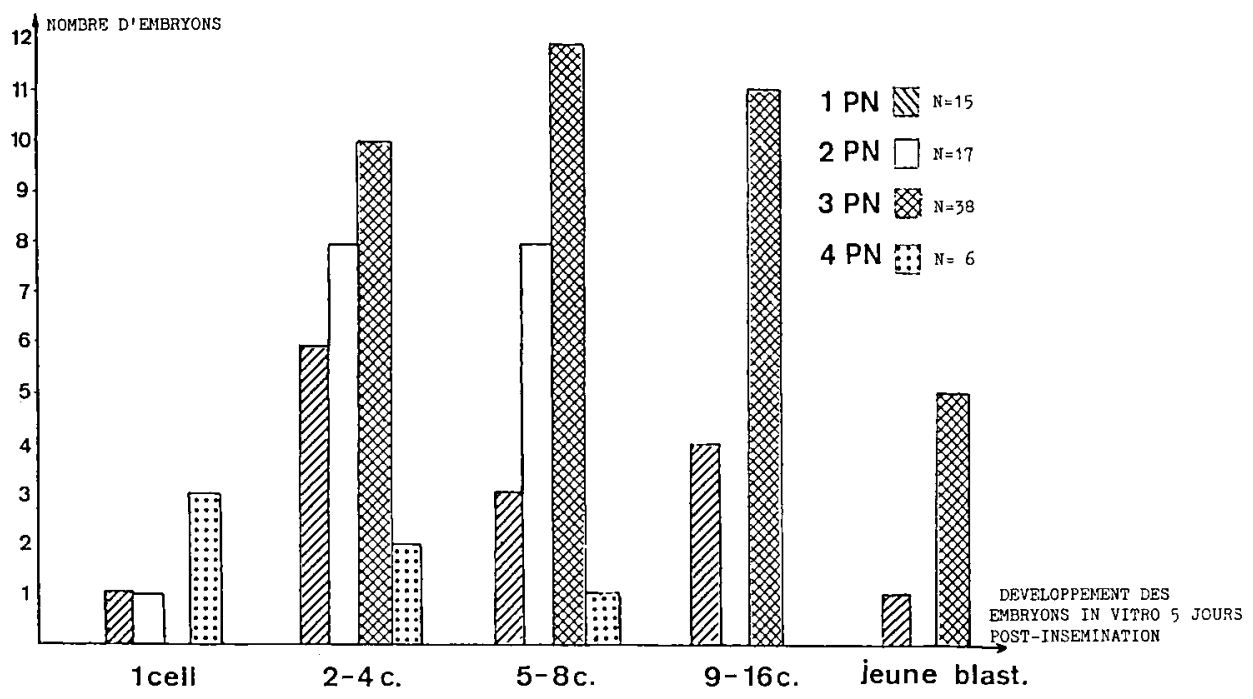

Les embryons provenant d'œufs à 4 pronuclei expriment une létalité précoce puisque aucun ne parvient au stade de morula.

Pour conclure, les embryons expriment très tôt leurs différences chromosomiques par des différences phénotypiques. Si les différences sont discrètes entre parthénogenèse et œufs diploïdes, les triploïdes évoluent différemment. Ils représentent un modèle idéal d'étude du développement embryonnaire précoce, de ses régulations et de ses anomalies d'autant plus qu'ils sont disponibles pour la recherche à une époque où l'embryon humain normal expérimental n'existe plus.

$27^{e}$ Réunion de la Société française pour l'Etude de la Fertilité. Paris, 29, 30 sept., $\gamma^{\text {er }}$ oct. 1988.

\section{Références}

BARTON S. C., SURANI M. A. H., NORRIS M. L., 1984. Role of paternal and maternal genomes in mouse development. Nature, 311, 374.

BUSTER J. E., BUSILLO M., RODI I. A., COHEN S. W., HAMILTON M., SIMON J. A., THORNEYCROFT I. H., MARSHALL S. R., 1985. Biologic and morphologic development of donated human ova recovered by non-surgical uterine lavage. Am. J. Obstet. Gynecol., 153, 211-217.

HERTIG A. T., ROCK J., ADAMS E. C., 1956. A description of human ova within the first 17 day of development. Am. J. Anat., 98, 435.

MARTIN R. H., MAHADEVAN M. M., TAYLOR P. J., HILDEBRAND K., LONG-SIMPSON L., PETERSON D., YAMAMOTO J., FLEETHAM J., 1986. Chromosomal analysis of unfertilized human oocytes. J. Reprod. Fert., 78, 673-678. 
PEREDA J., CROXATTO H. B., 1978. Ultrastructure of a seven cell human embryo. Biol Reprod., 18, $481-489$.

PLACHOT M., MANDELBAUM J., JUNCA A. M., COHEN J., SALAT-BAROUX J., DA LAGE C., 1987. Morphologic and Cytologic study of human embryos obtained by in vitro fertilization. In FEICHTINGER W., KEMETER P., Future aspects in human in vitro fertilization. Springer-Verlag, pp. 267-275.

PLACHOT M., JUNCAA. M., MANDELBAUM J., COHEN J., SALAT-BAROUX J., 1988a. Anomalies chromosomiques et FIV : Les limites du phénotype embryonnaire. Contracept. Fertil. Sex. 16. 648-651.

PLACHOT M., de GROUCHY J., JUNCA A. M., MANDELBAUM J., SALAT-BAROUX J., COHEN J., 1988b. Chromosome analysis of human oocytes and embryos : does delayed fertilization increase chromosome imbalance ? Hum. Reprod., 3, 125-127.

PLACHOT M., de GROUCHY J., JUNCA A. M., MANDELBAUM J., COHEN J., SALAT-BAROUX J., VEIGA A., CALDERON G., SANTALO J., BARRI P., EGOZCUE J., MONTAGUT J., LEPRETRE S., CARLES E., DEGOY J., SABATIER J. C., 1988c. Are clinical and biological IVF parameters correlated with chromosomal disorders in early life : a multicentric study. Hum. Reprod., 3, 627-635.

SAUER M. V., BUSTILLO M., RODI I. A., GORRILL M. J., BUSTER J. E., 1987. In vivo blastocyst production and ovum yield among fertile women. Hum. Reprod., 2, 701-703.

SHALGI R., 1984. Developmental capacity of rat embryos produced by in vivo or in vitro fertilization. Gam. Res., 10, 77-82.

TROUNSON A., SATHANANTHAN A. H., 1984. The application of electron microscopy in the evaluation of two - to four cell human embryos cultured in vitro for embryo transfer. J. IVF, $1,153$.

WRAMSBY H., FREDGA K., LIEDHOLM P., 1987. Chromosome analysis of human oocytes recovered from preovulatory follicles in stimulated cycles. N. Engl. J. Med., 316, 121-124. 